\title{
Design of coupled cavity with energy modulated electron cyclotron resonance ion source for materials irradiation research
}

\author{
Z. Wang (王智), ${ }^{1, *}$ J. E. Chen (陈佳洱), ${ }^{1}$ M. L. Kang (康明否) ${ }^{2}$ Y. R. Lu (陆元荣), ${ }^{1}$ W. L. Xia (夏文龙) ${ }^{1}$ \\ S. L. Gao (高淑丽), ${ }^{1}$ Z. Y. Guo (郭之虞), ${ }^{1}$ G. Liu (刘戈), ${ }^{1}$ S. X. Peng (彭士香), ${ }^{1}$ H. T. Ren (任海涛), ${ }^{1}$ \\ X. Q. Yan (颜学庆), ${ }^{1}$ J. Zhao (赵捷), ${ }^{1}$ and K. Zhu (朱昆) ${ }^{1}$ \\ ${ }^{1}$ State Key Laboratory of Nuclear Physics and Technology, Institute of Heavy Ion Physics, Peking University, Beijing 100871, China \\ ${ }^{2}$ Department of Radiation Oncology, Cancer Institute and Hospital, Chinese Academy of Medical Sciences, Beijing 100021, China
} (Received 10 November 2011; published 3 May 2012)

\begin{abstract}
The surface topography of samples after irradiation with heavy ions, protons, and helium ions based on accelerators is an important issue in the study of materials irradiation. We have coupled the separated function radio frequency quadrupole (SFRFQ) electrodes and the traditional RFQ electrodes into a single cavity that can provide a $0.8 \mathrm{MeV}$ helium beam for our materials irradiation project. The higher accelerating efficiency has been verified by the successful commissioning of the prototype SFRFQ cavity. An energy modulated electron cyclotron resonance (ECR) ion source can achieve a well-bunched beam by loading a sine wave voltage onto the extracted electrodes. Bunching is achieved without the need for an external bunch cavity, which can substantially reduce the cost of the system and the length of the beam line. The coupled RFQ-SFRFQ with an energy modulated ECR ion source will lead to a more compact accelerator system. The conceptual design of this novel structure is presented in this paper.
\end{abstract}

DOI: 10.1103/PhysRevSTAB.15.050101

PACS numbers: 29.27.Bd

\section{INTRODUCTION}

There is ongoing interest in the use of accelerators to study irradiation effects on materials that are used in reactors [1,2]. Ionic irradiation significantly damages the surface of materials to which it is exposed, as is encountered in the interior of fusion vessels. By simultaneously implanting ions such as carbon, oxygen, hydrogen, and helium, it may be possible to unravel the complex microstructural and microchemical evolutions that are expected in advanced nuclear energy systems. An accelerator-based materials irradiation facility is planned that will be used in conjunction with theory, simulation, and modeling to address the challenges presented by fusion wall materials and to study the form of the resulting waste. The main purposes of the materials irradiation facility are the detailed investigations of (1) the coupled electronic and atomic dynamics in the evolution of ion-beam damage, including the combined effects of electronic and nuclear stopping; and (2) the evolution of microstructural, physical, and chemical properties of complex actinide fuels and nuclear waste management materials.

Because of the simultaneous transverse focusing and longitudinal bunching in a cavity, a radio frequency quadrupole (RFQ) accelerator is considered to be the most suitable linear accelerator for the acceleration of low

\footnotetext{
*wangzhi@pku.edu.cn
}

Published by the American Physical Society under the terms of the Creative Commons Attribution 3.0 License. Further distribution of this work must maintain attribution to the author(s) and the published article's title, journal citation, and DOI. energy and high current beams [3,4]. However, a long RFQ cavity will decrease the efficiency of rf power. The ISAC-RFQ is a unique accelerator that operates in the $\mathrm{cw}$ mode for heavier ions [5,6]. Because the accelerating field is provided only by vane modulation, it requires a length of 8 meters to accelerate the heavy ions to $150 \mathrm{keV} / \mathrm{u}$ at a constant $-25^{\circ}$ accelerating phase. To avoid such a long accelerator, many types of $\beta \lambda / 2$-type postaccelerators have been developed, such as the hybrid RFQ $[7,8]$ and the rf focused interdigital (RFI) [9] accelerator. For the same reason, the separated function radio frequency quadrupole (SFRFQ) structure was proposed by the RFQ group of the Institute of Heavy Ion Physics at Peking University [10]. The SFRFQ structure combines the characteristics of the conventional RFQ and drift tube linac structures. It consists of a series of accelerating gaps and standard quadrupole lenses that are formed by loading diaphragms onto the unmodulated minivane quadrupole electrodes [11-13]. Based on studies of the SFRFQ dynamics and the space charge dominated beam [14-16], the SFRFQCODEV1.0 code [17] was specially developed for beam dynamics simulations and error analysis of the SFRFQ structure. The commissioning of the prototype cavity has verified that higher accelerating efficiency is obtained by introducing accelerating gaps. At the same time, transverse stability is ensured through the careful selection of the transverse focusing parameters. To obtain high transmission, only wellbunched beams from the RFQ should be accelerated by the SFRFQ. Based on the experience gained while working on the integral split ring radio frequency quadrupole (ISR-RFQ) $1000[18,19]$ and during the commissioning of the prototype SFRFQ cavity [20], advances in the 
engineering of key components of the resonant cavity have been made [21]. Because the RFQ and SFRFQ electrodes can both be excited by the split rings, they can be coupled and excited inside a single cavity. By replacing the accelerating section of the RFQ with the SFRFQ structure, the length of the RFQ accelerator and the cost of the linac can be substantially reduced. A new linac will couple the RFQ and SFRFQ structures resonating in one cavity, and the RFQ section will be used as a connection between the ion source and the SFRFQ structure. This coupled cavity will be developed as a compact accelerator complex to provide a $\mathrm{He}^{+}$ion beam for our project.

\section{DESIGN CONSIDERATIONS}

\section{A. Ion source and low energy beam transport (LEBT)}

To further accelerate the beam, the SFRFQ requires well-bunched beams from the RFQ. However, it is very difficult to generate the ideal longitudinal emittance directly from the ion source when using a $\mathrm{cw}$ input beam. Generally, an external harmonic buncher with a quasilinear bunching field can generate a small longitudinal emittance beam at the exit of the RFQ [22,23]. In our earlier work, we developed an electron cyclotron resonance (ECR) ion source by loading a sine wave voltage onto the extracted electrodes, for which the voltage amplitude was $1 \%-3 \%$ of the extracted voltage. This applied voltage can modulate the energy of beam from the ECR ion source and generate a bunched beam for the RFQ. The properties of the resulting beam were similar to the beam obtained using a buncher before the RFQ. This method is attractive because (1) the extracted beam from the ion source can be bunched without the need for rf cavities and (2) the entire system can be more compact. The main parameters of the energy modulated ECR ion source are listed in Table I.

Figure 1 shows the schematic layout of the LEBT for the coupled cavity. It consists of two solenoids and two sets of steering devices. There are two considerations in the design of the LEBT. First, because the charged ion beam is transported with space compensation induced by the ionization of gases such as $\mathrm{Ar}, \mathrm{Kr}$, or $\mathrm{N}_{2}$, the extracted beam

TABLE I. Parameters of the helium injector for the coupled RFQ-SFRFQ cavity.

\begin{tabular}{lc}
\hline \hline Particles & ${ }^{4} \mathrm{He}^{+}$ \\
Current (mA) & 20 \\
Particle energy (keV) & 30 \\
Macropulse frequency (Hz) & $166-\mathrm{cw}$ \\
Pulse width (ms) & 1.0 \\
Phase width (deg) & 120 \\
Duty factor & $1 / 6-\mathrm{cw}$ \\
$\varepsilon_{\text {unorm,rms, } x, y}(\mathrm{~mm} \mathrm{mrad})$ & 37.313 \\
$\varepsilon_{\text {norm,rms, } x, y}(\mathrm{~mm} \mathrm{mrad})$ & 0.1497 \\
$\alpha$ & 1.447 \\
$\beta(\mathrm{cm} / \mathrm{rad})$ & 7.7064 \\
\hline \hline
\end{tabular}

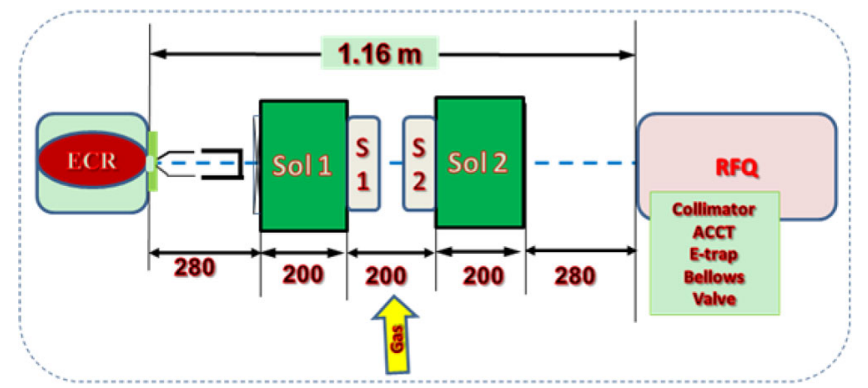

FIG. 1. The schematic view of helium injector.

from the LEBT to the entrance of the RFQ may include other high $Z$ ions. As a result, these ions will hit the inner wall of the solenoids and will be lost before entering the RFQ. The solenoids can provide high purity beams while preserving the matched beams for the RFQ. The second consideration is that the LEBT produce an adequate scope for the future beam tests.

If we apply a sine wave voltage to the extracted electrodes of the ion source, the energy of the extracted beam will be modulated as follows:

$$
\begin{gathered}
d w=q V_{m} T \sin (\omega t) \\
\text { focal length: } F=\frac{\beta \lambda}{\pi R_{m}},
\end{gathered}
$$

where $V_{m}$ is the amplitude of the modulated voltage, $T$ is the transit time factor of the extracted electrodes, $R_{m}$ is the ratio of the modulated voltage to the extracted voltage, $f$ is the frequency of the sine wave, and $\omega=2 \pi f$.

LMOVE is a time-energy phase-space dynamics program that calculates the evolution of an ion beam from an ion source with a particular mass, charge, energy, and time position in the longitudinal phase space. When the energy of the ion beam is modulated as described by Eq. (1), the extracted beam will be bunched after a length of drift space.

The simulation results for different ratios of extracted voltage from $1 \%-3 \%$ at the frequency of $26 \mathrm{MHz}(\beta \lambda=$ $0.053 \mathrm{~m}, e_{0}=0.1 \%$, where $e_{0}$ is the original energy spread of the unmodulated beam) are listed in Table II.

For $R_{m}=1 \%$, the extracted voltage of the ECR ion source is $30 \mathrm{kV}$, and the evolution of the modulated beam in the longitudinal phase space by LMOVE is shown in Fig. 2. Figure 2(d) shows that the extracted beam from the ECR ion source is bunched in the longitudinal phase space after a drift length of $L=F$. This result shows that this method can achieve a good bunched beam, which

TABLE II. Longitudinal phase width for different ratios of extracted voltage.

\begin{tabular}{lll}
\hline \hline$R_{m}$ & $F(\mathrm{~m})$ & $\tau(\mathrm{ns})$ \\
\hline $1 \%$ & 1.70 & 1.22 \\
$2 \%$ & 0.85 & 0.61 \\
$3 \%$ & 0.57 & 0.41 \\
\hline \hline
\end{tabular}


(a)

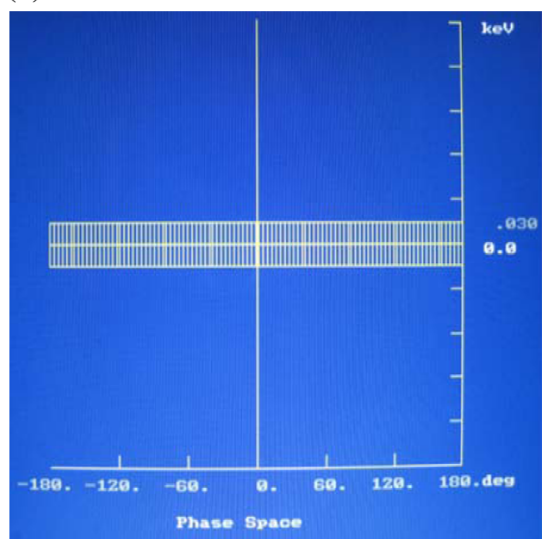

(c)

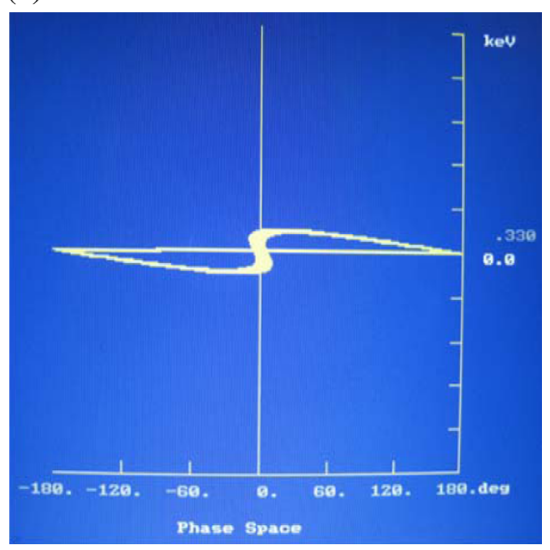

(b)

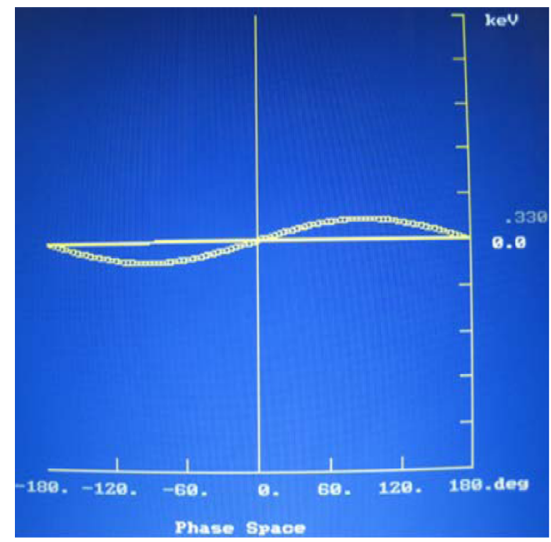

(d)

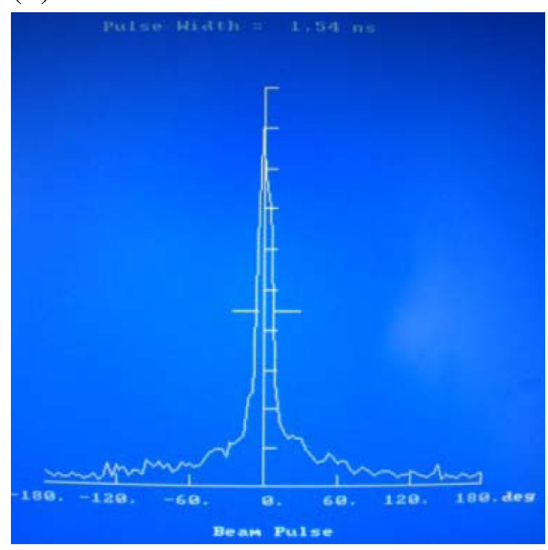

FIG. 2. Evolution of the modulated beam in the longitudinal phase plane. (a) Initial phase-energy distribution from ion source with energy spread of $0.1 \%$. (b) Phase energy of the extracted beam after introducing a voltage modulation. (c) Phase energy at $L=F$. (d) Beam pulse at $L=F$.

would be used as a quasibuncher before the RFQ in our project.

\section{B. Matching position between RFQ and SFRFQ}

In the first design stage, the longitudinal matching and the optimum matching position for the coupled

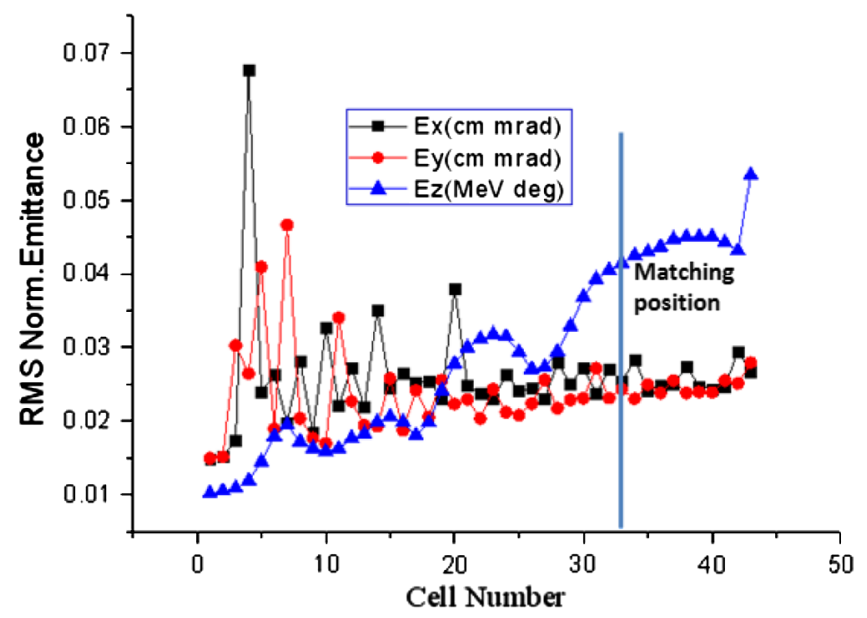

FIG. 3. Emittance variations along the RFQ cell number.
RFQ-SFRFQ cavity were considered. The choice of the RFQ-SFRFQ transition energy is a trade-off between adequate output energy from the RFQ and an acceptable longitudinal emittance for the SFRFQ. From the calculations of PARMTEQM, the longitudinal emittance along the RFQ is plotted in Fig. 3. To accomplish the longitudinal phase space matching between the RFQ and the SFRFQ, only 33 RFQ cells are used in the coupled RFQ-SFRFQ cavity.

In our definition, synchronous phase $\phi_{s}=-30^{\circ}$ in cell 33 of the RFQ means that the reference particle arrives at the center of cell 33 , which is characterized by an accelerating field proportional to $\cos (\omega t)$, when $\omega t=\phi_{s}=$ $-30^{\circ}$. Table III shows an example for $\phi_{s}=-30^{\circ}$ [24].

TABLE III. Phases in cell 33 for $\phi_{s}=-30^{\circ}$ of PARMTEQM program.

\begin{tabular}{lllll}
\hline \hline Convention & $\begin{array}{c}\text { Start } \\
\text { of cell }\end{array}$ & $\begin{array}{l}\text { Center } \\
\text { of cell }\end{array}$ & $\begin{array}{c}\text { End } \\
\text { of cell }\end{array}$ & $\begin{array}{c}\text { Start } \\
\text { next cell }\end{array}$ \\
\hline Cosine & -120 & -30 & +60 & -120 \\
Sine & -30 & +60 & +150 & -30 \\
\hline \hline
\end{tabular}




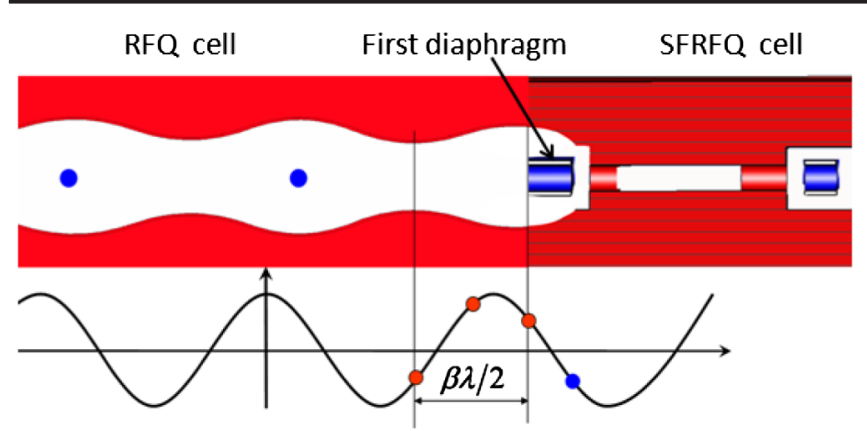

FIG. 4. Cross section ( $x-z$ plane of the coupled RFQ-SFRFQ (the three red points and one blue point in the cosine curve refer to the evolution of the synchronous phase).

When the reference particle arrives at the end of the cell, the phase in reference to a cosine is $+60^{\circ}$. At this point, the PARMTEQM code subtracts $180^{\circ}$ before starting consideration of the next cell.

SFRFQCODEV1.0 is intended specifically for the simulation of the beam dynamics of the SFRFQ structure. The synchronous phase used for cavity design refers to a cosine wave. Figure 4 shows that the phase is $60^{\circ}$ at the last cell of the RFQ, and the synchronous phase at the first accelerating gap of the SFRFQ is approximately $111^{\circ}$. Consequently, a negative accelerating field is required to achieve an energy gain. At the same time, this matching method utilizes the focusing and defocusing strengths provided by the rf quadrupole field to choose the proper length of the diaphragms after the accelerating gaps in each cell.

\section{DESIGN STUDY AND ERROR ANALYSIS}

The planned coupled RFQ-SFRFQ cavity will accelerate the $\mathrm{He}^{+}$beam to approximately $0.8 \mathrm{MeV}$ with a current of $5 \mathrm{~mA}$. The cavity consists of $33 \mathrm{RFQ}$ cells and 10 SFRFQ cells. The basic parameters of the coupled RFQ-SFRFQ cavity are listed in Table IV.

TABLE IV. Parameters of the coupled RFQ-SFRFQ cavity.

\begin{tabular}{lc}
\hline \hline Operating frequency/MHz & 26.0 \\
Charge to mass ratio & $\geq 1 / 4$ \\
Duty factor & $1 / 6$ \\
Intervane voltage $(\mathrm{kV})$ & 65 \\
Synchronous phase $(\mathrm{deg})$ & $69--30$ \\
Input energy of RFQ $(\mathrm{keV} / \mathrm{u})$ & 7.5 \\
Output energy RFQ $(\mathrm{keV} / \mathrm{u})$ & 105.7 \\
Length of RFQ $(\mathrm{cm})$ & 145.9 \\
Input energy of SFRFQ $(\mathrm{keV} / \mathrm{u})$ & 105.7 \\
Output energy SFRFQ $(\mathrm{keV} / \mathrm{u})$ & 201.2 \\
Length of SFRFQ $(\mathrm{cm})$ & 97.95 \\
Total length $(\mathrm{cm})$ & 243.8 \\
\hline \hline
\end{tabular}

The resonating frequencies of the RFQ and SFRFQ were adjusted to the $26 \mathrm{MHz}$ by changing the distance between rings of the RFQ section and the SFRFQ section, respectively. A 3D illustration of the coupled section is shown in Fig. 5. We see that the distance between the supporting rings in the side of the RFQ (Ls) was greater than that of the SFRFQ (Lr). From Fig. 6 we know that 4 diaphragms will be installed in one SFRFQ cell, which enhances the capacitance of the SFRFQ. To balance the capacitance and inductance, the distance between the two adjacent supporting rings of the SFRFQ was reduced.

A simulation of the coupled cavity has been performed using the CST MWS code [25]. The simulation results show that the two types of electrodes can be abutted directly and couple well with the same supporting rings in a single cavity. However, because of the introduction of the diaphragms, the specific shunt impedance of the coupled RFQ-SFRFQ was lower than that of a conventional RFQ accelerator of the same length by approximately $20 \%$. The surface current distribution on the supporting rings was uniform, as shown in Fig. 7.

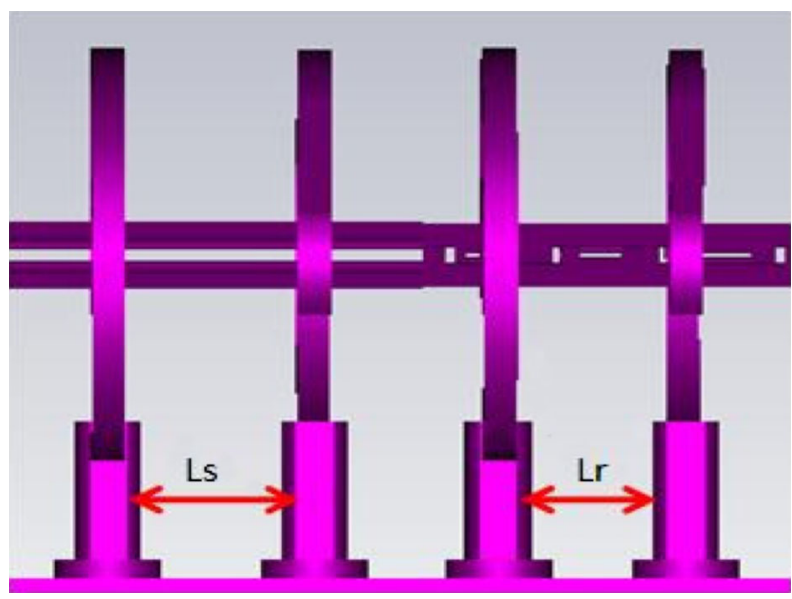

FIG. 5. The 3D illustration of the coupled section.

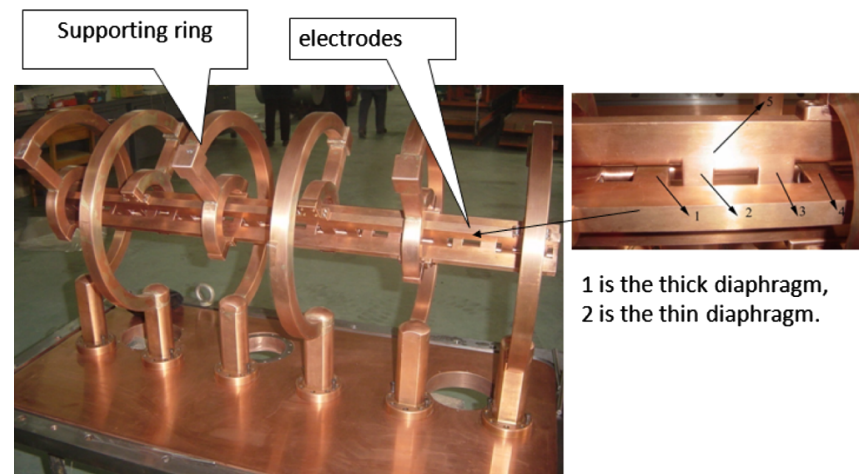

FIG. 6. SFRFQ structure with diaphragms loading on quadrupole poles. 


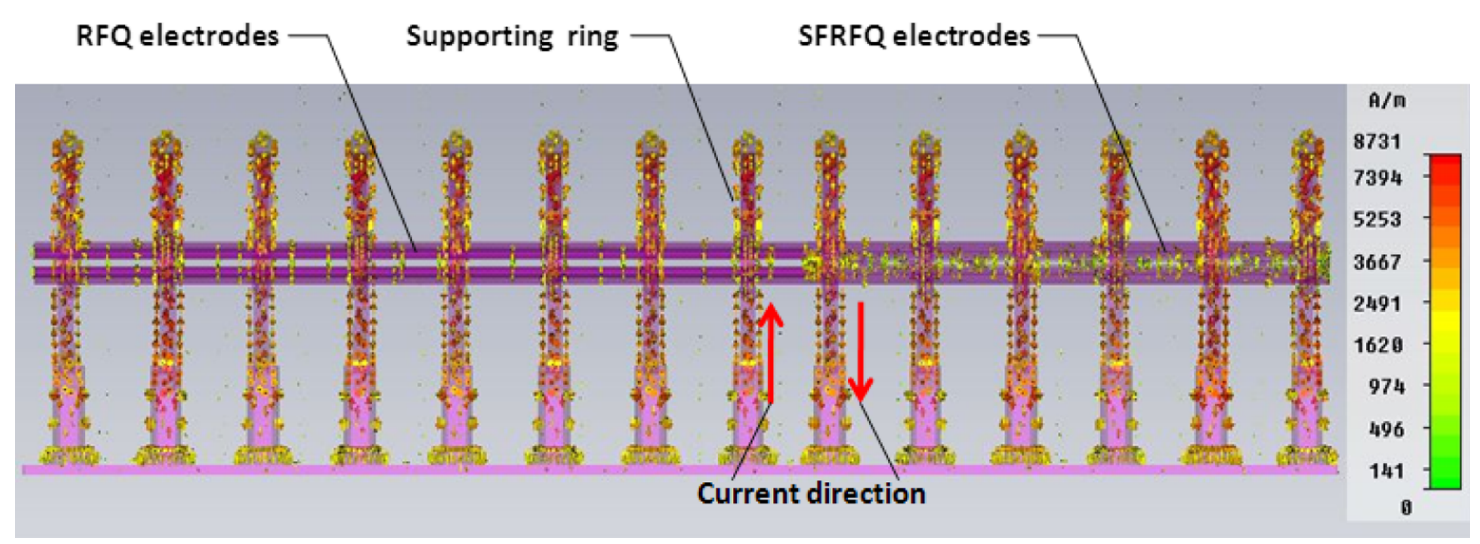

FIG. 7. Surface current distribution of the coupled cavity.

TABLE V. Ellipse parameters at the end of the 33rd cell.

\begin{tabular}{lcccc}
\hline \hline & $\alpha \mathrm{cm} / \mathrm{rad}$ & $\beta \mathrm{cmmrad}$ & $\begin{array}{c}\text { Emit, } u, \mathrm{rms} \\
\mathrm{cm} \mathrm{mrad}\end{array}$ & $\begin{array}{c}\text { Emit, } n, \mathrm{rms} \\
\mathrm{cm} \mathrm{mrad}\end{array}$ \\
\hline$x:$ & 2.1641 & 21.9684 & 1.8320 & 0.0276 \\
$y:$ & -1.5856 & 9.3170 & 1.4961 & 0.0225 \\
& & $\mathrm{deg} / \mathrm{MeV}$ & $\mathrm{MeV} \mathrm{deg}$ & $\mathrm{MeV} \mathrm{deg}$ \\
$z:$ & 2715.8380 & 0.0508 & 0.0508 \\
\hline \hline
\end{tabular}
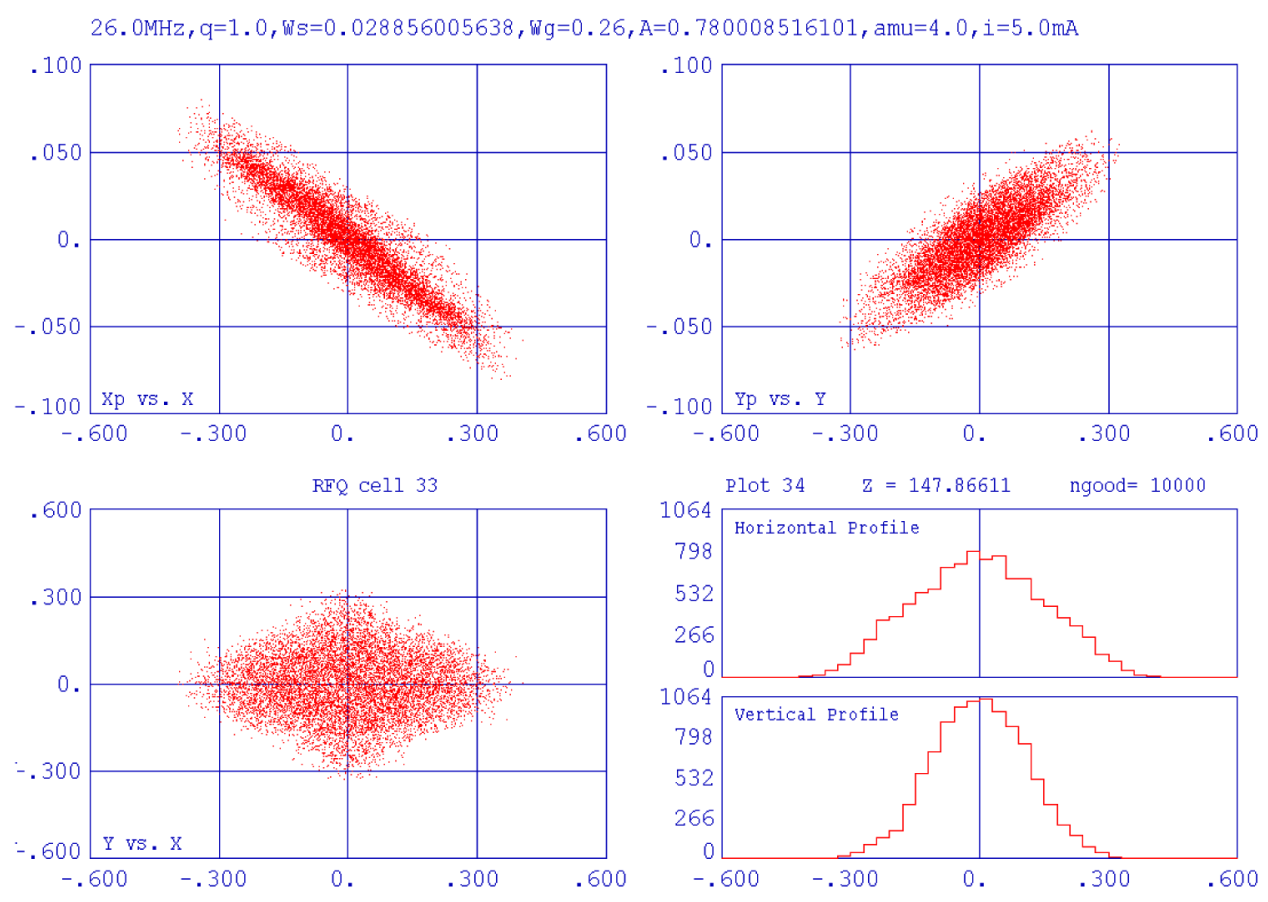

FIG. 8. Output beam distributions at the end of the 33rd cell of the RFQ. The output beam distribution in phase space and the optimized RFQ parameters such as the modulation $m$ and the synchronous phase $\phi_{s}$, are plotted in Figs. 8 and 9 , respectively. 


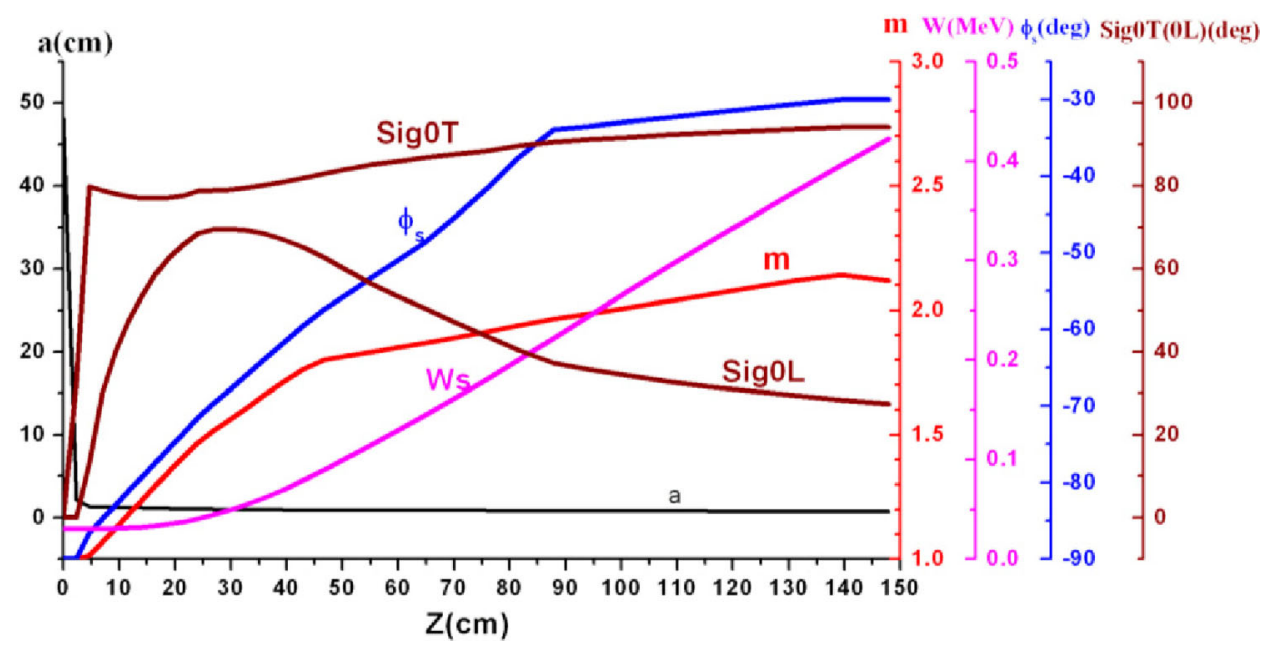

FIG. 9. Main parameters of the RFQ.

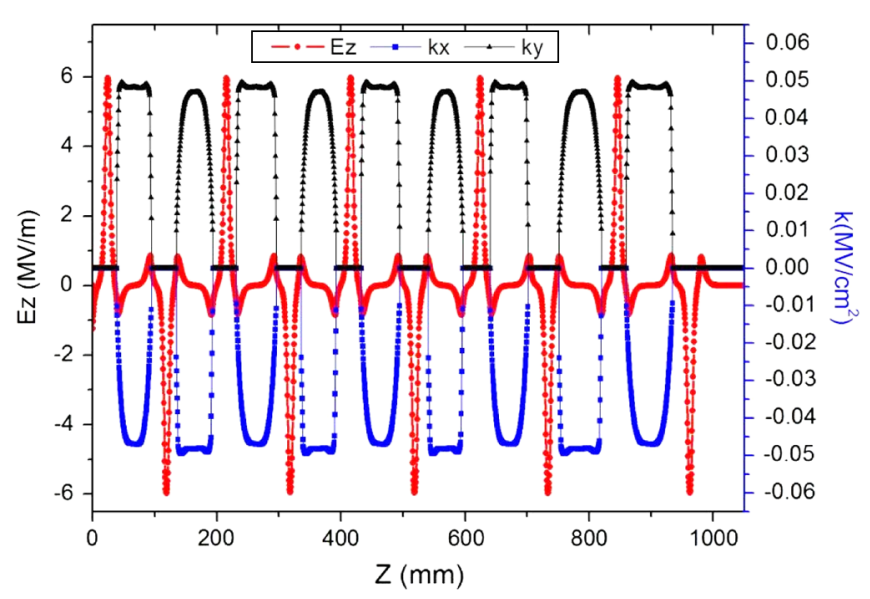

FIG. 10. The focusing and accelerating field distribution along the SFRFQ.

\section{A. RFQ section}

A conventional RFQ adiabatically bunches the beam to achieve good longitudinal acceptance. Because of the injection of a continuous beam, considerable length is required to form a beam bunch in the longitudinal direction. Generally, the modulation index $(\mathrm{m})$ increases very slowly. The design parameters of our first RFQ can be found in Ref. [13]. The energy modulated ECR ion source produces a pulsed input beam instead of a continuous beam for the RFQ. Therefore, the acceleration starts immediately after the radial matching section ( 2 cells), and the modulation index $(m)$ increases quickly from 1.01 to 2.14 . This method can substantially reduce the RFQ length. The LANL-type design, as for our coupled cavity, would have resulted in RFQ electrodes in excess of $2 \mathrm{~m}$. The ellipse parameters at the end of the 33rd cell determined from the PARMTEQM simulations using 10000 macroparticles are listed in Table V.

\section{B. SFRFQ section}

The characteristics of the beam dynamics in the SFRFQ section depend strongly on an array of the length of the diaphragms. Significant effort was put into the optimization of the length of the quadrupole field in each cell.

To achieve adequate transverse focusing strength, we determined that the thick and thin diaphragms should be

TABLE VI. Diaphragm parameters of the SFRFQ.

\begin{tabular}{lcccc}
\hline \hline $\begin{array}{c}\text { Cell } \\
\text { number }\end{array}$ & $\begin{array}{c}\text { Cell } \\
\text { length }(\mathrm{mm})\end{array}$ & $\begin{array}{c}\text { Thick diaphragm } \\
(\mathrm{mm})\end{array}$ & $\begin{array}{c}\text { Thin diaphragm } \\
(\mathrm{mm})\end{array}$ & $\begin{array}{c}\text { Ratio of quadrupole field } \\
\text { length to one cell }\end{array}$ \\
\hline 1 & 94.78 & 20 & 11 & $58.9 \%$ \\
2 & 96.78 & 20 & 12 & $58.7 \%$ \\
3 & 104.45 & 18 & 12 & $63.0 \%$ \\
4 & 98.40 & 18 & 14 & $59.0 \%$ \\
5 & 102.50 & 19 & 13 & $61.2 \%$ \\
6 & 106.20 & 20 & 18 & $56.4 \%$ \\
7 & 108.75 & 24 & 12 & $59.7 \%$ \\
8 & 111.95 & 28 & 14 & $55.5 \%$ \\
9 & 115.71 & 22 & 10 & $65.6 \%$ \\
10 & 40.01 & 24 & 12 & No focusing \\
\hline \hline
\end{tabular}




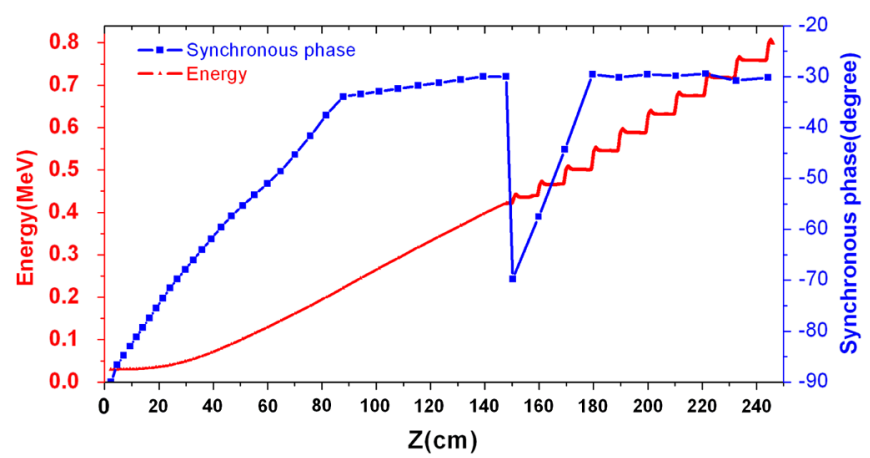

FIG. 11. Synchronous phase-cell number curve of coupled RFQ-SFRFQ cavity (subtracts $180^{\circ}$ at the end of each cell).

alternated. Both the length of the unit cell and the diaphragm length varied because of the energy gain of the beam. The results of a simulation of the accelerating field and transversal focusing field by SFRFQCODEV1.0 are presented in Fig. 10. The detailed lengths of the cell and diaphragms are listed in Table VI.

Figure 11 shows the evolution of the synchronous phase along the coupled cavity. The synchronous phase is approximately $-90^{\circ}$ at the beginning of the RFQ. It increases to $-30^{\circ}$ at the end of the RFQ cell. The first thin diaphragm, designed to avoid beam loss, causes a jump in the first synchronous phase of the SFRFQ section $\left(-69^{\circ}\right)$.

The transverse and longitudinal beam envelopes of the SFRFQ calculated using SFRFQCODEV1.0 are shown in Fig. 12. The statistical results plotted in Fig. 13 indicate that the main losses occur in the last 3 cells.

Figure 14 shows the full energy spread at the exit of the coupled RFQ-SFRFQ cavity. The final energy of the beam reaches its designed value, and the full energy spread is less than 3.5\%. The region for inspection was 400-600 nm in depth from the irradiation surface, where the ratio of the injected helium concentration to the displacements per

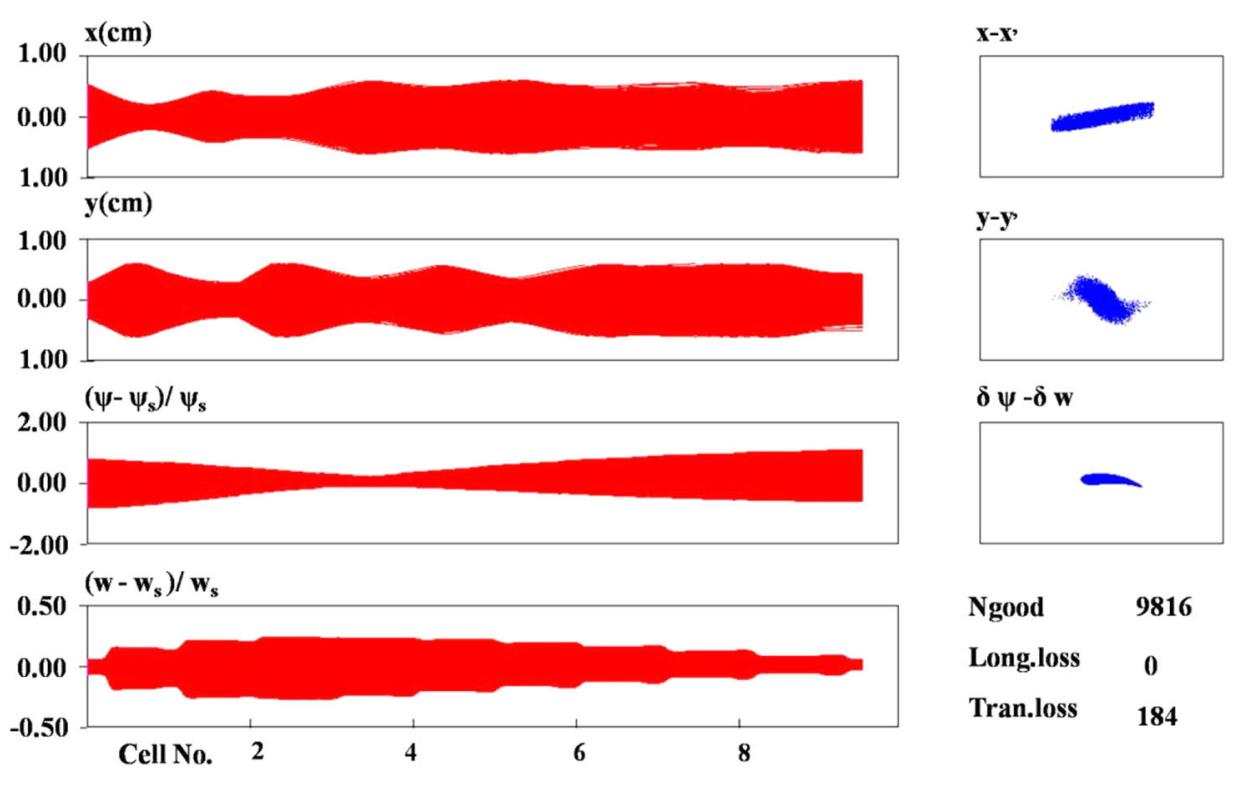

FIG. 12. Transverse and longitudinal beam envelope in SFRFQ.

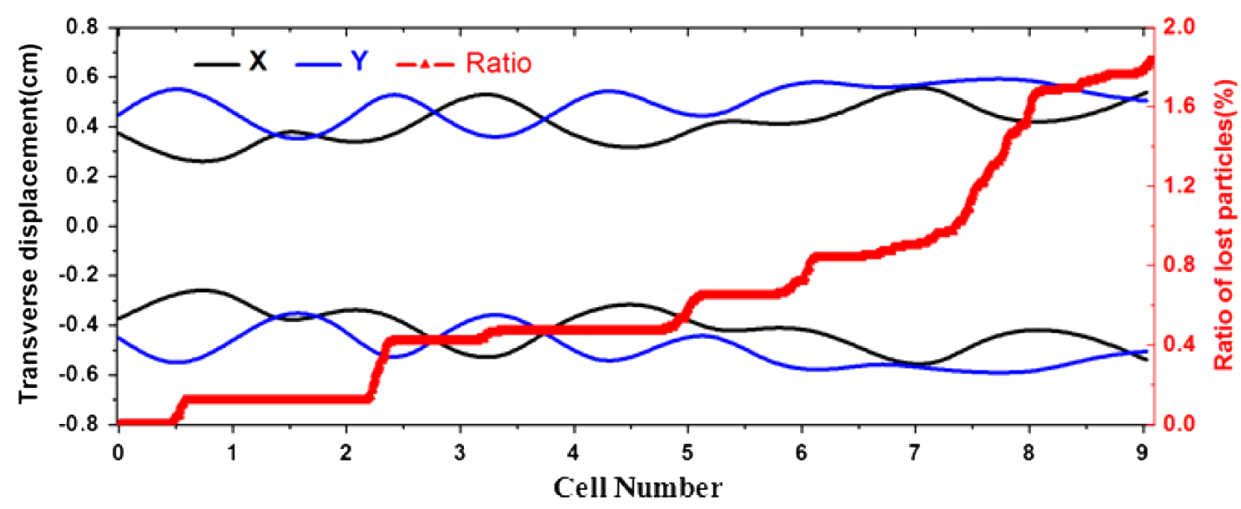

FIG. 13. Ratio of lost particles to total particles. 

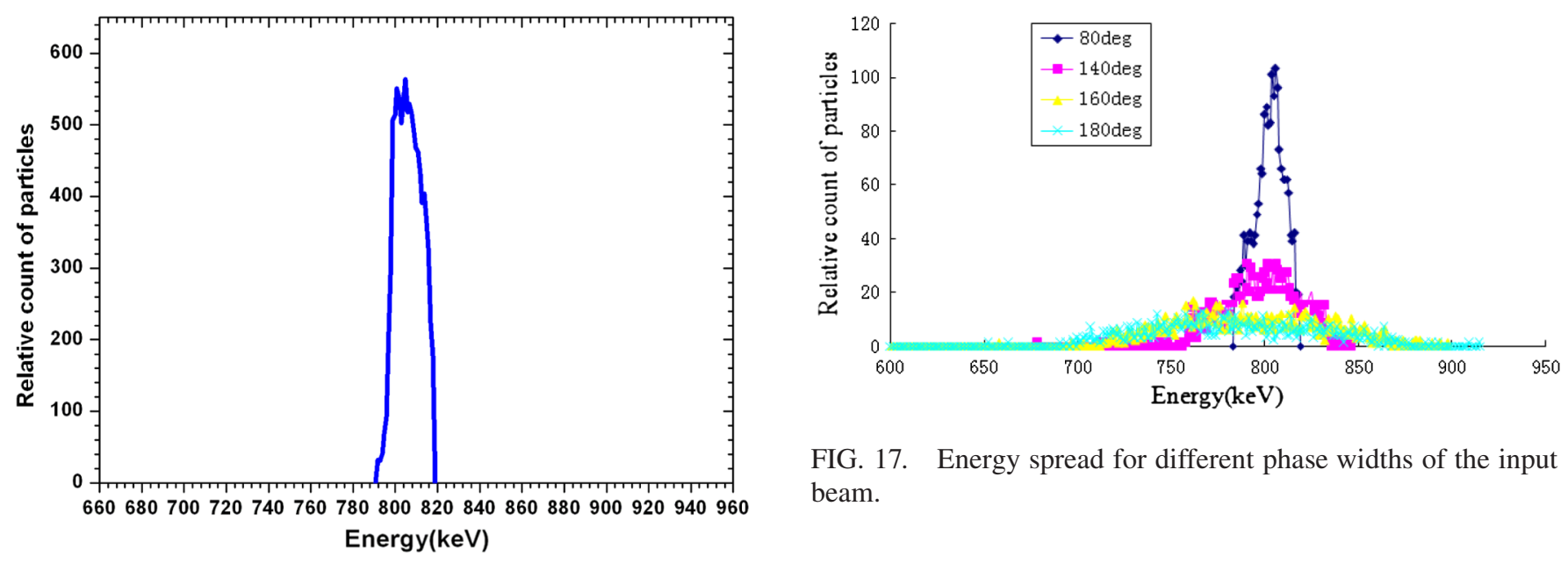

FIG. 17. Energy spread for different phase widths of the input beam.

FIG. 14. Energy spectrum of the coupled RFQ-SFRFQ cavity.
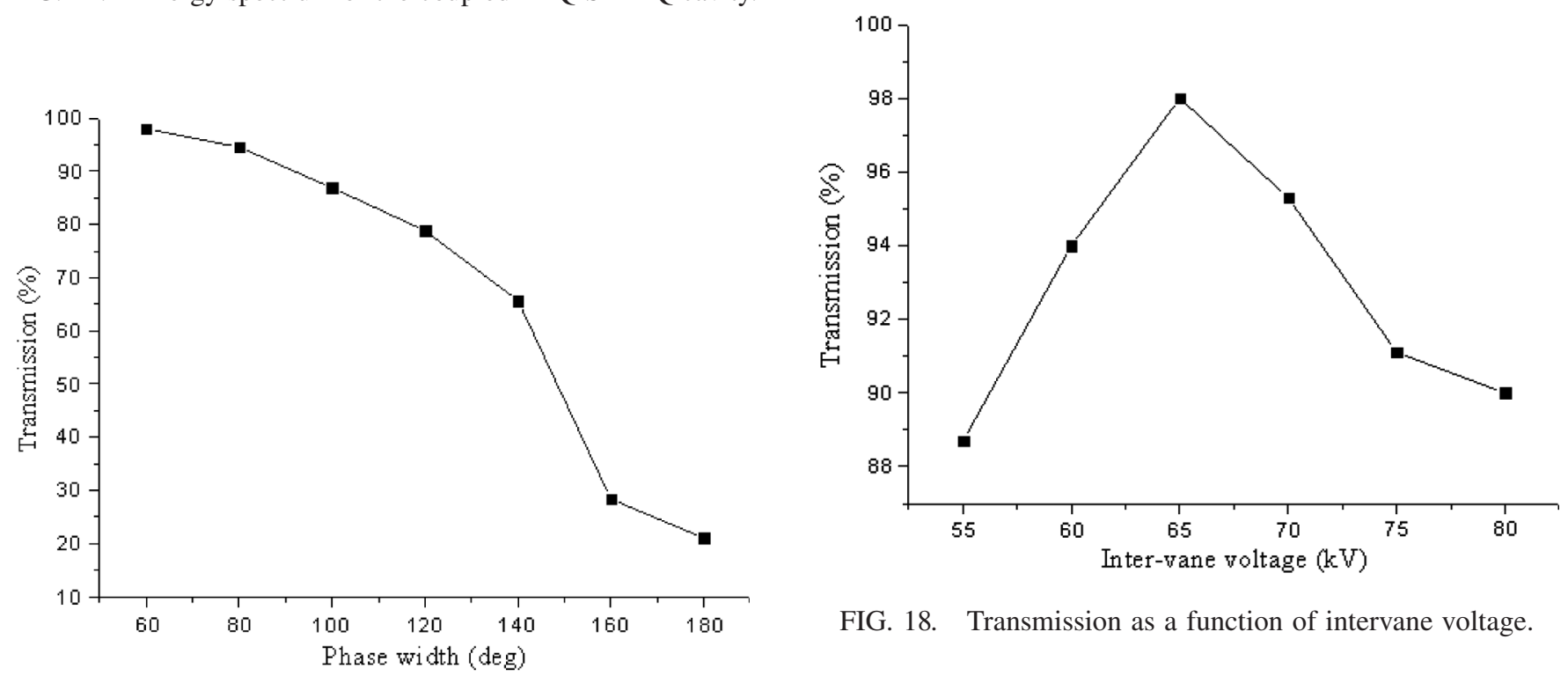

FIG. 18. Transmission as a function of intervane voltage.

FIG. 15. Transmission for different phase widths of the input beam.

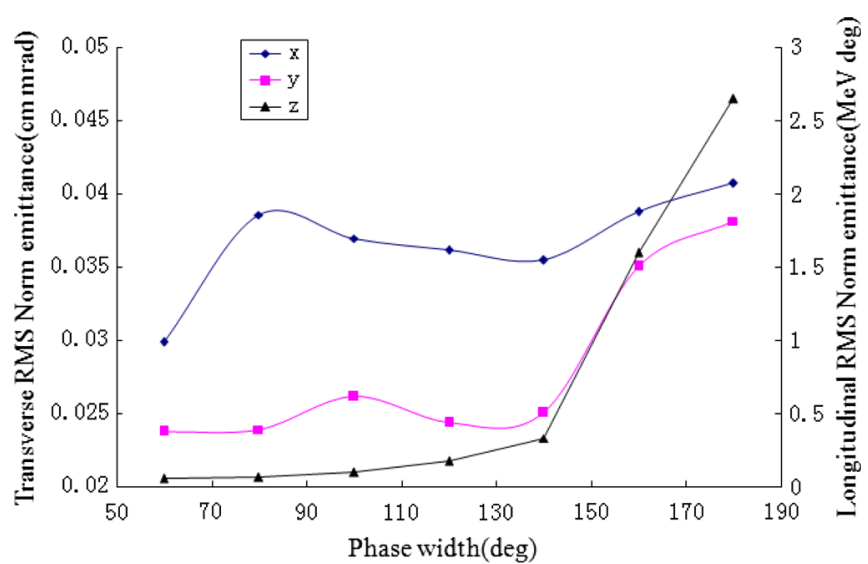

FIG. 16. Emittance of the output beam as a function of the phase width.

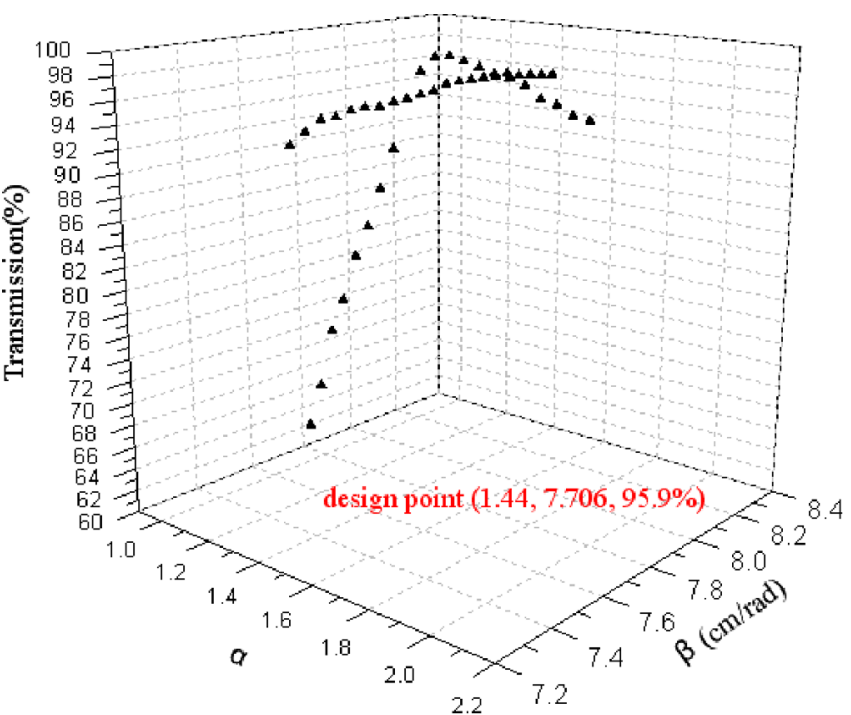

FIG. 19. Transmission as a function of transverse beam ellipse parameters. 
TABLE VII. Characteristics of different rf structures.

\begin{tabular}{lccccc}
\hline \hline & $\begin{array}{c}\text { ISAC-RFQ } \\
{[5,6]}\end{array}$ & $\begin{array}{c}\text { Hybrid } \\
\text { RFQ [7,8]] }\end{array}$ & $\begin{array}{c}\text { Coupled } \\
\text { RFQ-RFI [9] }\end{array}$ & $\begin{array}{c}\text { Coupled } \\
\text { RFQ-SFRFQ } \\
\text { of this paper }\end{array}$ & $\begin{array}{c}\text { Coupled } \\
\text { RFQ-SFRFQ } \\
\text { (optimized) }\end{array}$ \\
\hline Bunched input beam & Yes & Yes & No & Yes & Yes \\
Prebuncher cavity & Yes & Yes & No & No & No \\
$A / q$ & 30 & 238 & 4 & 4 & 4 \\
$F(\mathrm{MHz})$ & 35 & 12 & 200 & 26 & 104 \\
Intervane voltage $(\mathrm{kV})$ & 75 & 100 & & 65 & 70 \\
Length $(\mathrm{m})$ & 8 & 3.34 & 2.2 & 2.5 & $5-412.5$ \\
Energy in-out $(\mathrm{keV} / \mathrm{u})$ & $2-150$ & $7.12-20.61$ & $25-2500$ & $7.5-201.2$ & 407.5 \\
Energy gain $(\mathrm{keV} / \mathrm{u})$ & 148 & 13.49 & 2475 & 193.7 & 1.63 \\
Voltage gain $(\mathrm{MV})$ & 4.44 & 3.211 & 2.475 & 0.778 & 0.815 \\
Gradient Ea $(\mathrm{MV} / \mathrm{m})$ & 0.555 & 0.96 & 1.125 & 0.31 & 26.5 (calculated) \\
rf power $(\mathrm{kW} / \mathrm{m})$ & 9.4 & 3.6 & 55.9 & 12 & \\
\hline \hline
\end{tabular}

atom (dpa), He/dpa, was approximately 15 , and the ion fluence rate reached more than $1.5 \times 10^{12} \mathrm{~cm}^{-2} \mathrm{~s}^{-1}$.

\section{Error analysis}

The properties of the beam extracted from the ECR ion source are an important consideration for the entire cavity. Because parameters such as transversal emittance and beam current are easy to control, the most important parameter is the phase width. Figures 15 and 16 show the transmission and emittance as a function of the input beam phase width at the entrance of the RFQ.

A large longitudinal phase width may lead to particles that are lost or not accelerated synchronously by the SFRFQ. To find the lowest acceptable value of the phase width, the energy spectrum obtained using various input beam phase widths are compared in Fig. 17. We found that the energy spectrum deteriorates when the phase width exceeds $\pm 80^{\circ}$.

The intervane voltage of the coupled RFQ-SFRFQ may change because of the rf amplifier instability. This affects the synchronous phase and changes the particle energy gain. This effect may cause transmission variation, as shown in Fig. 18.

The transverse beam quality at the entrance of the RFQ has a significant influence on the transmission of the entire cavity. The transmission is plotted as a function of the beam ellipse parameters $\alpha$ and $\beta$ in Fig. 19.

\section{CONCLUSIONS AND OUTLOOK}

Based on our experience with ISR-RFQ 1000 and the commissioning of the prototype SFRFQ cavity, we have developed a novel structure that couples RFQ and SFRFQ electrodes in a single cavity for materials irradiation research. The coupled RFQ-SFRFQ structure can improve the accelerating efficiency by replacing the accelerating section in the RFQ with an SFRFQ structure. We have carried out the concept design of the coupled RFQ-SFRFQ with an energy modulated ECR ion source that can compress the continuous beam into a bunched beam without requiring prebunchers before the RFQ. Most RFQs require an external buncher to reduce the entire cavity length and improve accelerating efficiency. However, this can be costly. Our method, loading a sine wave voltage onto the extracted electrodes of an ion source, delivers a wellbunched beam without requiring an external bunch cavity, substantially reducing the cost of the system.

The coupled RFQ-SFRFQ adopted a lower frequency $(26 \mathrm{MHz})$ to accelerate $\mathrm{He}^{+}$ions because there was not a higher frequency rf power source. Table VII lists the characteristics of different $\mathrm{rf}$ structures. It shows that the accelerating gradient $\mathrm{Ea}(\mathrm{MV} / \mathrm{m})$ of our RFQ-SFRFQ design is $0.31 \mathrm{MV} / \mathrm{m}$, which is lower than the gradients of the ISACRFQ and the hybrid RFQ. By increasing the resonating frequency to $104 \mathrm{MHz}$, the cavity length of the existing design was reduced. The optimized cavity consists of 131 RFQ cells and 22 SFRFQ cells. The RFQ section will accelerate the $\mathrm{He}^{+}$ions to $0.7 \mathrm{MeV}$. The SFRFQ section will further accelerate the beam to $1.65 \mathrm{MeV}$. The total length of the optimized cavity is approximately $2 \mathrm{~m}$. The accelerating gradient $\mathrm{Ea}(\mathrm{MV} / \mathrm{m})$ will reach $0.815 \mathrm{MV} / \mathrm{m}$.

Further studies will be needed to address the high duty factor operation. In the first step, through more experience with the operation of the coupled RFQ-SFRFQ cavity, we will develop a reliable and easy-to-maintain accelerator complex in our lab to provide the $\mathrm{He}^{+}$beam for materials irradiation experiments. In the second step, we will develop a coupled RFQ-SFRFQ operated with an energy modulated ion source that will work at a higher frequency. Consequently, the accelerating efficiency of the coupled RFQ-SFRFQ will be significantly improved.

\section{ACKNOWLEDGMENTS}

Many thanks to Professor J. Q. Lv for the permission to use the LMOVE computer code, which has been used for the simulations of the extracted beam from the energy 
modulated ECR ion source to the entrance of the coupled RFQ-SFRFQ cavity in longitudinal phase space. This work was supported by the National Natural Science Foundation of China (Grant No. 10905003, Grant No. 11079001, and No. 91026012) and China Postdoctoral Science Foundation.

[1] T. Muroga, M. Gasparotto, and S. J. Zinkle, Fusion Eng. Des. 61, 13 (2002).

[2] R. A. Jameson et al., Nucl. Instrum. Methods Phys. Res., Sect. B 68, 474 (1992).

[3] I. Kapchinsky and V. A. Teplyakov, Prib. Tekh. Eksp. 2, 19 (1970).

[4] R.H. Stokes et al., IEEE Trans. Nucl. Sci. 26, 3469 (1979).

[5] R. E. Laxdal, R. A. Baartman, and L. Root, in Proceedings of the 18th Particle Accelerator Conference, New York, 1999 (IEEE, New York, 1999).

[6] R.L. Poirier et al., in Proceedings of the 20th International Linac Conference, Monterey, CA, 2000 (SLAC, Menlo Park, CA, 2000).

[7] P. N. Ostroumov, M. P. Kelly, and A. A. Kolomiets, Nucl. Instrum. Methods Phys. Res., Sect. B 204, 433 (2003).

[8] P. N. Ostroumov et al., Nucl. Instrum. Methods Phys. Res., Sect. A 547, 259 (2005).

[9] W. Joel Starling and Donald A. Swenson, Nucl. Instrum. Methods Phys. Res., Sect. B 261, 21 (2007).

[10] J.E. Chen et al., Prog. Nat. Sci. 12, 22 (2002).
[11] X. Q. Yan et al., Nucl. Instrum. Methods Phys. Res., Sect. A 506, 1 (2003).

[12] X. Q. Yan et al., Nucl. Instrum. Methods Phys. Res., Sect. A 539, 606 (2005).

[13] C. E. Chen et al., in Proceedings of the European Particle Accelerator Conference, Vienna, 2000 (EPS, Geneva, 2000).

[14] Leonid G. Vorobiev and Richard C. York, Phys. Rev. ST Accel. Beams 3, 114201 (2000).

[15] Oliver Boine-Frankenheim and Tripti Shukla, Phys. Rev. ST Accel. Beams 8, 034201 (2005).

[16] J. Amundson et al., J. Comput. Phys. 211, 229 (2006).

[17] Z. Wang et al., Nucl. Instrum. Methods Phys. Res., Sect. A 572, 596 (2007).

[18] Y. R. Lu et al., Nucl. Instrum. Methods Phys. Res., Sect. A 515, 394 (2003).

[19] X. Q. Yan et al., Phys. Rev. ST Accel. Beams 9, 020101 (2006).

[20] M. L. Kang et al., Nucl. Instrum. Methods Phys. Res., Sect. A 640, 38 (2011).

[21] Z. Wang et al., Nucl. Instrum. Methods Phys. Res., Sect. A 607, 522 (2009).

[22] Alok Chakrabarti et al., Nucl. Instrum. Methods Phys. Res., Sect. A 535, 599 (2004).

[23] Q. Zhao et al., in Proceedings of the Linear Accelerator Conference (JACOW, Lübeck, Germany, 2004), THP03.

[24] Manual of the LANL RFQ Design Codes, LANL Report No. LA-UR-96-1836, revised 2005, p. 49.

[25] http://www.cst.com. 